\title{
Stem cell therapy- An update
}

\author{
Fazlur Rahman1, Bikash Subedi1, Anisul Awal1, K.M.H.S. Sirajul Haque1, Md. Abu Siddique ${ }^{1}$ \\ Sajal Krishna Banerjee1, Md. Safiuddin'1, Chaudhury Meshkat Ahmed ${ }^{1}$, Syed Ali Ahsan ${ }^{1}$, Khairul \\ Anam 1 , Md. Khurshed Ahmed ${ }^{1}$, Md. Mukleshur Rahman¹, Md. Ashraf Uddin Sultan ${ }^{1}$ \\ Md. A. Rashid ${ }^{1}$ and Mohammad Salman. ${ }^{1}$ \\ ${ }^{1}$ Department of Cardiology, Bangabandhu Sheikh Mujib Medical University, Shahbag, Dhaka.
}

Address for Correspondence

Dr. Bikash Subedi, Department of Cardiology, BSMMU, Shahbag, Dhaka.

\begin{abstract}
Stem cells are one of the most fascinating areas of biology today. Stem cells have two important characteristics that distinguish them from other types of cells. First, they are unspecialized cells that renew themselves for long periods through cell division. The second is that under certain physiologic or experimental conditions, they can be induced to become cells with special functions. Stem cells can be obtained from several sources including spare embryos, special purposes embryos, aborted fetus, umbilical cords, adult tissue or organs, cadavers. The procedure of adult stem cell therapy involves severalsteps including extraction of cells from various sources, separation by surface markers using FACS, growth in specificculture media and injection into target organs. This modern aspect of regenerative or reparative medicine can be applied for cardiac diseases (coronary artery diseases, heart failure, non ischaemic cardiomyopathies), skin replacement, Diabetes, spinal cord injury, neurodegenerative disease like Parkinsonism, stroke. In future, it may become possible the use of stem cells in organ transplantation.
\end{abstract}

\section{Introduction}

Stem cells are the cells that have remarkable potential to develop into many different cell types in the body. Stem cells are one of the most fascinating areas of biology today. Serving as a sort of repair system for the body, they can theoretically divide without limit to replenish other cells for as long as the person or animal is still alive. When a stem cell divides, each "daughter" cell has the potential to either remain a stem cell or become another type of cell with a more specialized function, such as a muscle cell, a red blood cell, or a brain cell.

Stem cells have two important characteristics that distinguish them from other types of cells. First, they are unspecialized cells that renew themselves for long periods through cell division. The second is that under certain physiologic or experimental conditions, they can be induced to become cells with special functions such as the beating cells of the heart muscle or the insulin-producing cells of the pancreas.

Stem cells are important for living organism for many reasons. In the 3 to 5 days old embryo, called blastocyst, stem cells in developing tissues give rise to multiple specialized cell types that make up the heart, lung, skin and other tissues. In some adult tissues, such as bone marrow, muscle and brain, discrete population of generate replacement for cells that are lost through normal wear, tear, injury or disease. ${ }^{1}$

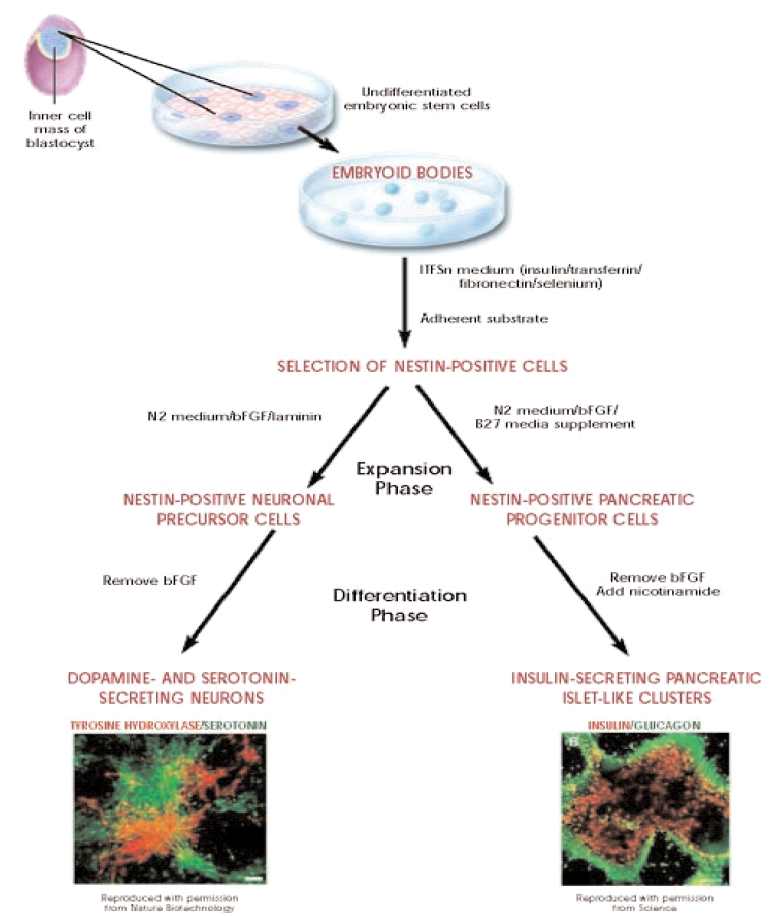

Figure : Differentiation of embryonic stem cell.

Scientists primarily work with 2 kinds of stem cells from animals and human; embryonic stem cells and adult stem cells, which have different functions and characteristics. They discovered ways to obtain or derive stem cells from early mouse embryos more than 20 years ago. Many years 
of detailed study of the biology of mouse stem cells led to discovery in 1998, of how to isolate stem cells from human embryos and grow the cells in the laboratory. These are called the human embryonic stem cells. The embryos used in these studies were created for the infertility purposes through in vitro fertilization procedures and when they are no longer needed for that purposes, they are donated for research with the informed consent of the donor.

Stem cells can be obtained from several sources including spare embryos, special purposes embryos, aborted fetus, umbilical cords, adult tissue or organs, cadavers. ${ }^{2}$

Both adult and embryonic stem cells have advantages and disadvantages. Embryonic stem cells have advantages, namely, flexible, immortal and easily available but disadvantages are ethical controversies and at odds with patients immune system. Adult stem cells have advantages of already somewhat specialized, immune hardy, flexible and mixed degree of availability. However, the disadvantages of adult stem cells include minimal quantity and genetically unsuitable. ${ }^{2}$

This modern aspect of regenerative or reparative medicine can be applied for cardiac diseases (coronary artery diseases, heart failure, non ischaemic cardiomyopathies), skin replacement, Diabetes, spinal cord injury, neurodegenerative disease like Parkinsonism, stroke. In future, it may become possible the use of stem cells in organ transplantation.

The procedure of adult stem cell therapy involves several steps. First, adult autologous cells eg, bone marrow, peripheral blood, skeletal myoblast are taken. Then stem cells are separated by surface markers using FACS (fluorescent activated cell sorter). These separated cells are grown in vitro using specific culture media with addition of specific growth factors which may or may not differentiate within media. These cells are then injected into target organs by various methods. ${ }^{3}$

The possible routes of cell therapy in heart includes intracoronary, balloon catheter, intravenous, transendocardial, intramyocardial. ${ }^{4}$

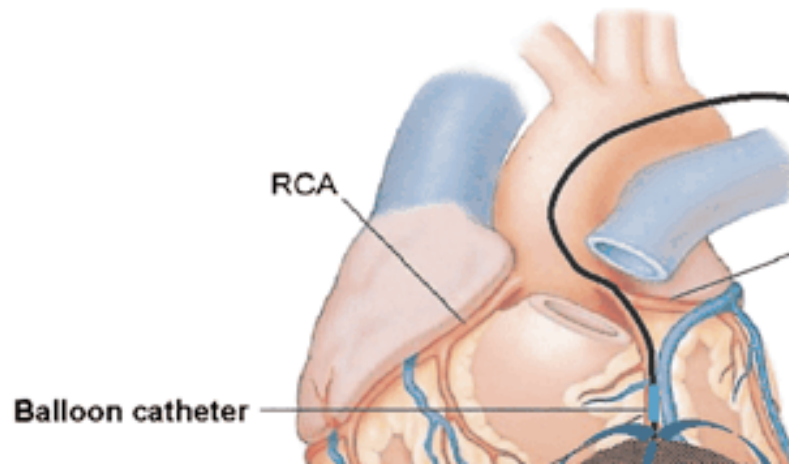

Figure : Routes for cell therapy in heart
After the injection in heart these cells by the virtue of specific chemokine receptor they reach the specific site of injury as the area of injury secrete chemokines. ${ }^{5}$

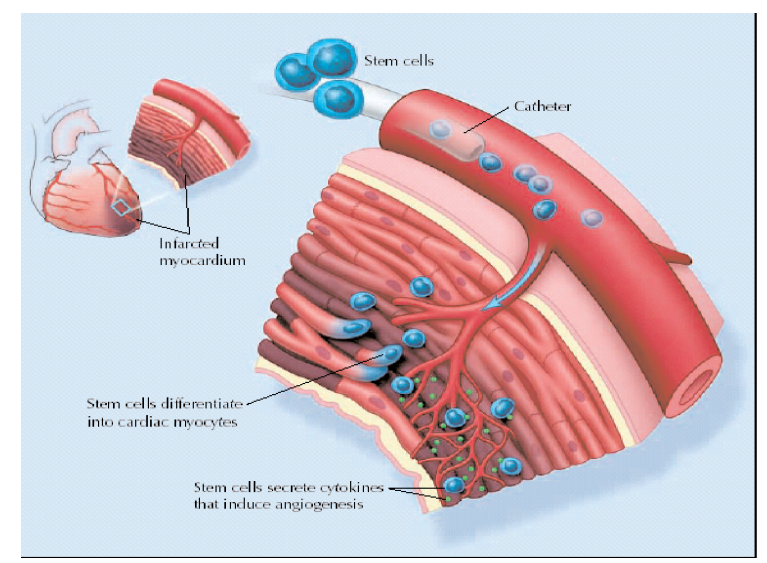

Figure : stem cells differentiating into cardiac myocyte and inducing angiogenesis

In contrast to our traditional view that damaged cardiac muscle are replaced by scar tissue, stem cell therapy has revolutionized the scenario with the replacement of damaged cardiac myocyte with the new ones. ${ }^{6}$

Stem cells differentiate into cardiac myocyte and also secrete the cytokines that induce angiogenesis, hence contributing to both angiogenesis and myogenesis. ${ }^{7}$

\section{Hurdles to come}

Although general enthusiasm for stem cell therapy for cardiac disease is fuelling intense research, there are potential adverse effects of this novel treatment. For example, in patients who received intracoronary treatment, higher rates of restenosis in the stented culprit lesions in the coronary artery have been noted. Interestingly, the degree of neointimal growth was proportional to that of improvement in cardiac function. Although restenosis was successfully managed with the additional deployment of drug-eluting stents, this served as our first warning. Evidence from current trial, MAGIC Cell-III, showed that the timing of stem cell mobilization and the use of drug-eluting stents might solve the problem of restenosis caused by neointimal growth. To proceed with and improve stem cell therapy, we need a better understanding of stem cell biology. Areas of investigation include the optimal type of cell and the kind of modification necessary, whether a heterogeneous cell population is preferable to a purified one, or differentiated cells to undifferentiated cells, the best mobilization protocol and ways to overcome the barriers to the effective homing of stem cells to the harsh environment of infarcted tissue. 


\section{A promising new modality}

Currently, 2 biologic therapies for cardiovascular diseases - cell and gene therapy - are under investigation in clinical trials. In general, gene therapy has had limited success to date because of the short clinical effects observed and problems around the safety of the vector encoding new genetic material (typically viruses). However, gene therapy may hold the key to the future of stem cell therapy: by permitting controlled augmentation, proliferation and differentiation of stem cells through genetic manipulation, the current problems associated with stem cell therapy may be solved. Indeed, the potential benefits of stem cell therapy may not be fully realized until the advances of these seemingly divergent fields are united and give rise to an entirely new therapeutic modality. 8

\section{Future of cell therapy?}

Despite the many challenges, most scientists believe that cell therapy will revolutionize medicine. With the use of cell therapies, we may soon have dramatic cures for cancer, Parkinson's, diabetes, kidney disease, multiple sclerosis, muscular degeneration and a host of other diseases. Cell therapies have also shown great promise in helping to repair catastrophic spinal injuries, and helping victims of paralysis regain movement. It is even possible that the human life span could be greatly extended due to the replenishment of tissues in aging organs. We may even have the ability one day to grow our own organs for transplantation from our own stem cells, eliminating the danger of organ rejection. While we will undoubtedly encounter the limits of cell therapy one day, there is every reason to hope that this revolutionary new approach will result in radically improved ways to treat diseases.
To conclude; remaining young at heart is a desirable but elusive goal. Myocyte regeneration may accomplish just that. Continuous cell renewal in adult myocardium was thought to be impossible; multipotent stem cells may be able to renew myocardium and under certain circumstances, can be coaxed to repair the broken heart after infarction. ${ }^{9}$

\section{References}

1. Bethesda MD; National Institute health.U.S. Department of Health and Human Services, 2006

2. Lauren Pecorino: Stem cells for cell based therapies. www.actionbioscience.com,2008

3. Nicholas A Boon, Nicki R Colledge, Brian R Walker. John AA Hunter. Stem Cell therapy .Davidson's principle and practice of medicine $20^{\text {th }}$ edition. 2006.

4. Straucer BE, Kornowski R. Stem Cell Therapy in Perspectives.Circulation 2003:107:929-34

5. Rosenthal N. Promethesus's Vulture and the stem cell promise.N Engl j Med: 2003:349:267-74

6 Grounds MD et al. The role of stem cells in skeletal and cardiac muscle repair. J Histochem Cytochem. 2002;50:589-610

7. Dimmeler S et al. Cell Based Therapy of myocardial Infarction. Arterioscler Thromb Vasc Biol. 2007; 19

8. Hyun J Kang, Hyo SK, Young BP. Stem Cell Therapy for Myocardial Infarction Journal of Canadian Medical Association.2004;171(5)

9. Anversa P, Nadal-Ginard B et al.A matter of life and death: Cardiac myocyte apoptosis and death. Nature. 2002;415:240-43 